\title{
QUANTUM STOCHASTIC INTEGRALS AS BELATED INTEGRALS
}

\author{
by CHRIS BARNETT, J. M. LINDSAY and IVAN F. WILDE
}

(Received 19 November, 1990)

Introduction. Quantum stochastic integrals have been constructed in various contexts $[2,3,4,5,9]$ by adapting the construction of the classical $L^{2}$-Itô-integral with respect to Brownian motion. Thus, the integral is first defined for simple integrands as a finite sum, then one establishes certain isometry relations or suitable bounds to allow the extension, by continuity, to more general integrands. The integrator is typically operator-valued, the integrand is vector-valued or operator-valued and the quantum stochastic integral is then given as a vector in a Hilbert space, or as an operator on the Hilbert space determined by its action on suitable vectors.

These constructions are functional-analytic in essence rather than measure-theoretic. That stochastic integration can be thought of in measure-theoretic terms was established in [6]. Indeed, generalizing Bartle's construction of the bilinear vector integral [7] by replacing the semi-variation of the vector measure by the "semi-variation with respect to a filtration" (called the belated semi-variation) it was shown in [6] that one can define genuine vector integrals of adapted integrands. The quantum stochastic integrals of $[3,4$, 5] and, indeed, the clasical $L^{2}$-Itô-integral with respect to Brownian motion are special cases of these "belated integrals".

We shall show here that, by a further slight generalization (allowing a more general pairing between integrand and integrator) the Fock space quantum stochastic integrals of $[1,2,10]$ (namely those whose integrators are the fermion or boson annihilation, creation or number operator processes) can also be obtained as belated integrals.

In view of the "equivalence" between the fermion and boson cases [11], it is enough to treat just one or the other. We shall consider the latter.

1. The belated semivariation. We recall the relevant concepts from [6]. Let $\mathscr{R}$ denote the ring of subsets of $\mathbf{R}^{+}=[0, \infty)$ generated by bounded intervals, and let $m$ be a finitely-additive vector measure on $\mathscr{R}$ with values in a linear space $\mathscr{Y}$. Suppose that $\mathscr{X}$ is a normed space and let $\left\{\mathscr{X}_{s}\right\}_{s \in \mathbf{R}^{+}}$be an increasing family of subspaces of $\mathscr{X}$ indexed by $\mathbf{R}^{+}$. Let $\mathscr{Z}$ be a Banach space and suppose that there is a bilinear mapping from $\mathscr{X} \times \mathscr{Y}$ into $\mathscr{Z}$, $(x, y) \mapsto x y, x \in \mathscr{X}, y \in \mathscr{Y}$.

Definition 1.1. The belated semivariation of $m$ (with respect to $\left\{\mathscr{Q}_{s}\right\}$ ) is the set function

$$
E \mapsto\|E\|_{m}^{b}=\sup \left\|\sum x_{i} m\left(I_{i}\right)\right\| \quad(E \in \mathscr{R}),
$$

where the supremum is over all partitions of $E$ into a finite number of disjoint intervals $\left\{I_{i}\right\}$ and all $x_{i} \in \mathscr{X}_{\text {inf } l_{i}}$ with $\left\|x_{i}\right\| \leq 1$.

If $\mathscr{X}_{s}=\mathscr{X}$ for all $s \in \mathbf{R}^{+}$, then the belated semivariation reduces to the semivariation of $m[7]$.

The belated semivariation can be suitably extended when it has a control measure $\gamma$; that is, when there is a locally finite positive measure $\gamma$ such that, for any $t>0$, 
$\gamma(E) \rightarrow 0 \Rightarrow\|E\|_{m}^{b} \rightarrow 0$ for $E \in \mathscr{R}, E \subseteq[0, t]$. First $\|\cdot\|_{m}^{b}$ is extended to outer sets by using monotone sequences from $\mathscr{R}$ : if $F$ is an outer set with $F=\lim E_{n}$ where $\left(E_{n}\right)$ is an increasing sequence in $\mathscr{R}$, we define $\|F\|_{m}^{b_{\sigma}}=\lim \left\|E_{n}\right\|_{m}^{b_{a}}$. The $\gamma$ continuity of $\|\cdot\|_{m}^{b}$ on $\mathscr{R}$ ensures that $\|F\|_{m}^{b_{o}}$ is well-defined. If $A \subset \mathbf{R}^{+}$, we set

$$
\|A\|_{m}^{b_{o}}=\inf \left\{\|F\|_{m}^{b_{\sigma}}: F \supseteq A\right\},
$$

where the infimum is taken over all outer sets $F$ containing $A$. Thus $\|\cdot\|_{m}^{b_{\sigma}}$ is (locally) continuous with respect to the outer measure induced by $\gamma$. As explained in [6, Section 2], extending $\|\cdot\|_{m}^{b_{\sigma}}$ in this way leads to a wider class of integrable functions than one would otherwise obtain.

The belated semivariations of the measures considered here will be shown to have control measures and so will be extended as above with no further comment.

Definition 1.2. A map $\phi: \mathbf{R}^{+} \rightarrow \mathscr{X}$ is said to be a process (with respect to the filtration $\left.\left\{\mathscr{X}_{s}\right\}\right)$ if $\phi(s) \in \overline{\mathscr{X}}_{s}$, the closure of $\mathscr{X}_{s}$ in $\mathscr{X}$, for each $s \in \mathbf{R}^{+}$.

A process is elementary if it has the form $\phi=x \chi_{I}$ for some bounded interval $I \subset \mathbf{R}^{+}$ and some $x \in \mathscr{X}_{\text {inf } l}$, where $\chi_{I}$ denotes the indicator function of the interval $I$.

A process is simple if it is a finite linear combination of elementary processes.

It was shown in [6] that Bartle's methods [7] can be adapted to develop an integration theory for processes using the belated semivariation rather than the semivariation of the vector measure $m$. We note that for the development in [6] it is not necessary that the pairing $x y$ be defined for all $x \in \mathscr{X}$ and all $y \in \mathscr{Y}$. All that is needed is that $x y$ is defined (and is bilinear) for $y$ of the form $y=m(I)$, where $I$ is a bounded interval in $\mathbf{R}^{+}$, and for $x \in \mathscr{X}_{\text {inf } f}$. We shall be concerned here with the case when $\mathscr{X}$ is a Hilbert space and $\mathscr{Y}$ is a set of (unbounded, closed) operators in $\mathscr{X}$.

Let $\mathscr{H}$ denote the symmetric Fock space over $L^{2}\left(\mathbf{R}^{+}\right)$, and for $f \in L^{2}\left(\mathbf{R}^{+}\right)$, let $\psi(f)$ denote the exponential vector

$$
\psi(f)=1 \oplus f \oplus \ldots \oplus(n !)^{-1 / 2} \underbrace{f \otimes \ldots \otimes f)}_{n \text { times }} \oplus \ldots
$$

For $g \in L^{2}\left(\mathbf{R}^{+}\right)$and $k \in L^{\infty}\left(\mathbf{R}^{+}\right)$, let $a^{+}(g)$ and $a^{-}(g)$ denote the boson creation and annihilation operators and let $a^{0}(k)$ denote the preservation (or gauge, or number) operator, all considered as closed operators on $\mathscr{H}$.

Thus

$$
\begin{aligned}
& a^{-}(g) \psi(f)=\langle g, f\rangle \psi(f) \\
& a^{0}(k) \psi(f)=\left.\frac{d}{d t} \psi\left(e^{t k} f\right)\right|_{t=0} \\
& a^{+}(g) \psi(f)=\left.\frac{d}{d t} \psi(f+t g)\right|_{t=0} .
\end{aligned}
$$

Each operator is the closure of its restriction to the linear span of the exponential vectors, $a^{ \pm}(g)$ are mutually adjoint and, when $k$ is real-valued, $a^{0}(k)$ is self-adjoint.

The relation (1.1) is the basis of the analysis of [10] and, of course, is the reason for introducing exponential vectors. 
For $s \in \mathbf{R}^{+}$, let $\mathscr{B}_{s}$ be the complex unital polynomial algebra generated by the operators $a^{-}(g), a^{+}(g)$ where $g$ runs over those elements of $L^{2}\left(\mathbf{R}^{+}\right)$which vanish outside $[0, s]$, and, for $f \in L^{2}\left(\mathbf{R}^{+}\right)$, set $\mathscr{X}_{s}(f)=\overline{\mathscr{B}} \psi(f)$ and $\mathscr{X}=\mathscr{H}$. Then $\mathscr{X}_{s}(f) \subseteq \mathscr{X}_{t}(f) \subset \mathscr{X}$ for $0 \leq s \leq t$.

Let $\mathscr{Y}_{ \pm}$denote the set of closed operators $\left\{a^{ \pm}(g): g \in L^{2}\left(\mathbf{R}^{+}\right)\right\}$and $\mathscr{Y}_{0}$ the set of closed operators $\left\{a^{0}(k): k \in L^{\infty}\left(\mathbf{R}^{+}\right)\right\}$. These become linear spaces if we define

$$
\begin{gathered}
a^{ \pm}\left(g_{1}\right)+a^{ \pm}\left(g_{2}\right)=a^{ \pm}\left(g_{1}+g_{2}\right), \quad a^{0}\left(k_{1}\right)+a^{0}\left(k_{2}\right)=a^{0}\left(k_{1}+k_{2}\right), \\
\lambda a^{+}(g)=a^{+}(\lambda g), \quad \lambda a^{0}(k)=a^{0}(\lambda k), \quad \lambda a^{-}(g)=a^{-}(\bar{\lambda} g)
\end{gathered}
$$

for $\lambda \in \mathbf{C}, k, k_{1}, k_{2} \in L^{\infty}\left(\mathbf{R}^{+}\right)$, and $g, g_{1}, g_{2} \in L^{2}\left(\mathbf{R}^{+}\right)$.

2. Measures associated with $a^{+}, a^{0}$ and $a^{-}$. Let $u, v \in L_{\mathrm{loc}}^{2}\left(\mathbf{R}^{+}\right)$and $w \in L_{\mathrm{loc}}^{\infty}\left(\mathbf{R}^{+}\right)$be given. For $E \in \mathscr{R}$ set $\mu(E)=a^{-}\left(u \chi_{E}\right), v(E)=a^{+}\left(v \chi_{E}\right)$ and $\xi(E)=a^{0}\left(\omega \chi_{E}\right)$. Evidently, $\mu$, $v$ and $\xi$ are finitely-additive measures taking values in $\mathscr{Y}_{+}, \mathscr{Y}_{-}$and $\mathscr{Y}_{0}$, respectively. If $E \subset[s, \infty)$, then $\mathscr{S}_{s}(f)$ lies within the domain of the operators $\mu(E), v(E)$ and $\xi(E)$, thus defining a pairing with values in $\mathscr{L}=\mathscr{H}$. Thus, as remarked in Section 1 , the analysis of [6] is applicable.

Henceforth, we will write the pairing in the order $(x, y) \mapsto y x$ for $y=\mu(E)$ (or $v(E)$, $\xi(E)) E \subset[s, \infty)$ and $x \in \mathscr{R}_{s}(f)$.

THEOREM 2.1. The belated semivariation of $\mu$ (with respect to $\left.\left\{\mathscr{X}_{s}(f)\right\}\right)$ is given by

$$
\|E\|_{\mu}^{b}=\int_{E}|u(s) f(s)| d s
$$

for $E \in \mathscr{R}$.

Proof. For given $E \in \mathscr{R}$, let $\left\{I_{j}\right\}$ be a finite partition of $E$ into disjoint intervals, and let $x_{j} \in \mathscr{D}_{\text {inf } l_{i}}(f)$ with $\left\|x_{j}\right\| \leq 1$. Then, using equation (1.1), we see that

$$
\begin{aligned}
\left\|\sum_{j} \mu\left(I_{j}\right) x_{j}\right\| & =\left\|\sum_{j}\left\langle u \chi_{I_{j}}, f\right\rangle x_{j}\right\| \\
& \leq \sum_{j} \int_{l_{j}}|u(s) f(s)|\left\|x_{j}\right\| d s \\
& \leq \int_{E}|u(s) f(s)| d s,
\end{aligned}
$$

giving $\|E\|_{\mu}^{b} \leq \int_{E}|u(s) f(s)| d s$.

On the other hand, letting $x_{j}=\alpha_{j} \psi^{\prime}(f)$, with $\alpha_{j} \in \mathbf{C},\left|\alpha_{j}\right| \leq 1, \psi^{\prime}(f)=\psi(f) /\|\psi(f)\|$, and noting that $\psi(f) \in \mathscr{X}_{s}(f)$ for each $s \geq 0$, we obtain

$$
\begin{aligned}
\|E\|_{\mu}^{b} & \geq\left\|\sum_{j} \mu\left(I_{j}\right) \alpha_{j} \psi^{\prime}(f)\right\| \\
& =\left\|\sum_{j}\left\langle u \chi_{l_{j}}, f\right\rangle \alpha_{j} \psi^{\prime}(f)\right\| \\
& =\left|\sum_{j} \int_{l_{j}} \alpha_{j} \overline{u(s)} f(s) d s\right|
\end{aligned}
$$

since $\left\|\psi^{\prime}(f)\right\|=1$. 
Taking the supremum over all such partitions $\left\{I_{j}\right\}$ of $E$ and all $\alpha_{j} \in \mathbf{C}$ with $\left|\alpha_{j}\right| \leq 1$ gives $\|E\|_{\mu}^{b} \geq \int_{E}|u(s) f(s)| d s$, and the result follows.

To estimate the belated semivariations of $v$ and $\xi$, we shall use the methods of [10].

THEOREM 2.2. The belated semivariations of $v$ and $\xi$ (with respect to $\left\{\mathscr{P}_{s}(f)\right\}$ ) satisfy

$$
\begin{gathered}
\|E\|_{v}^{b} \leq\left\{\int_{E} e^{t-s}|v(s)|^{2}\left(1+|f(s)|^{2}\right) d s\right\}^{1 / 2} \\
\|E\|_{\xi}^{b} \leq\left\{\int_{E} e^{t-s}|w(s)|^{2}\left(|f(s)|^{2}+|f(s)|^{4}\right) d s\right\}^{1 / 2}
\end{gathered}
$$

for $E \in \mathscr{R}$, where $t=\sup E$.

Thus $v f \in L_{\mathrm{loc}}^{2}\left(\mathbf{R}^{+}\right)$and $f \in L_{\mathrm{loc}}^{4}\left(\mathbf{R}^{+}\right)$are sufficient conditions for the belated semivariations of $v$ and $\xi$, respectively, to have control measures.

Proof. Let $E \in \mathscr{R}$ and let $\left\{I_{j}\right\}$ be a partition of $E$ into a finite number of intervals. Let $x_{j} \in \mathscr{L}_{\text {in } t_{j}}(f)$ with $\left\|x_{j}\right\| \leq 1$. Then, using the methods of [10, Theorem 4.3], we obtain

$$
\begin{aligned}
\left\|\sum_{j} v\left(I_{j}\right) x_{j}\right\|^{2} & =\left\|\sum_{j} a^{+}\left(v \chi_{I_{j}}\right) x_{j}\right\|^{2} \\
& \leq \sum_{j} \int_{i_{j}} e^{t-s}|v(s)|^{2}\left(1+|f(s)|^{2}\right)\left\|x_{j}\right\|^{2} d s \\
& \leq \int_{E} e^{t-s}|v(s)|^{2}\left(1+|f(s)|^{2}\right) d s
\end{aligned}
$$

where $t=\sup E$. This gives (2.2) and a similar argument gives (2.3).

Note that Theorems 2.1 and 2.2 exhibit control measures of the form $E \mapsto \int_{E} \phi(s) d s$ for $\mu, v$ and $\xi$, when $v f$ and $f^{2}$ are locally square-integrable, thus allowing their belated semivariations to be extended by continuity to outer sets as indicated in Section 1 . The inequalities 2.1-2.3 remain valid for the extensions $\|\cdot\|_{\mu}^{b_{o}},\|\cdot\|_{v}^{b_{o}}$ and $\|\cdot\|_{\xi}^{b_{o}}$, respectively, with $E$ replaced by any outer set $F$ in $[0, t]$, and hence any Lebesgue measurable set in $[0, t]$.

3. Integrable processes. We recall the definition of belated integrability [6].

Definition 3.1. Let $h: \mathbf{R}^{+} \rightarrow \mathscr{H}$ be an elementary process; $h=\chi_{1} x$ for some finite interval $I$ and $x \in \mathscr{X}_{\text {inf }}(f)$. The $\mu^{b}$-integral of $h$ is defined to be $\int d \mu^{b} h=\mu(I) x$. The $\mu^{b}$-integral of any simple process is then defined by linearity.

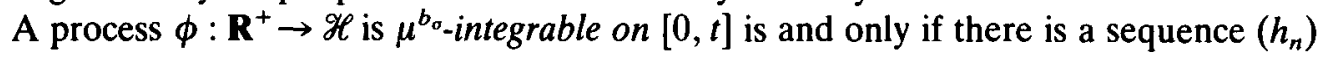
of simple processes such that

(i) $h_{n} \rightarrow \phi$ in $\mu^{b_{\sigma}}$-measure on $[0, t]$;

(ii) $\int_{E} d \mu^{b} h_{n} \equiv \int d \mu^{b} \chi_{E} h_{n} \rightarrow 0$ in $\mathscr{H}$ as $\|E\|_{\mu}^{b} \rightarrow 0$ with $E \in \mathscr{R}, E \subseteq[0, t]$, uniformly in $n$; i.e. $\left(h_{n}\right)$ is uniformly absolutely continuous with respect to $\mu^{b_{\sigma}}$ on $[0, t]$.

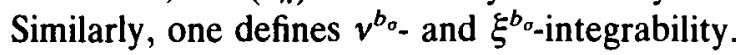

Remark. Condition (i) means that for any given $\epsilon>0$,

$$
\left\|\left\{s \in[0, t]:\left\|h_{n}(s)-\phi(s)\right\| \geq \epsilon\right\}\right\|_{\mu}^{b_{0}} \rightarrow 0
$$

as $n \rightarrow \infty$. 
We note that there is no third condition as in $[6,7]$. This is simply because $\|[0, t]\|_{\eta}^{b}$ is finite, for $\eta=\mu, v, \xi$.

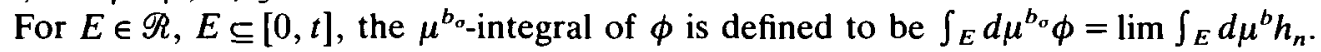
For completeness, we include a proof that this is well-defined. Similarly, one defines $\int_{E} d v^{b} o \phi$ and $\int_{E} d \xi^{b_{\sigma}} \phi$.

Theorem 3.2. Let $\phi$ be $\mu^{b_{\sigma}}$-integrable on $[0, t]$ and let $\left(h_{n}\right)$ be a sequence of simple processes as in Definition 3.1. Then, for any $E \in \mathscr{R}, E \subseteq[0, t],\left(\int_{E} d \mu^{b} h_{n}\right)$ is a Cauchy sequence in $\mathscr{H}$.

Proof. Let $E \in \mathscr{R}, E \subseteq[0, t]$ and $\epsilon>0$ be given. Let $\delta>0$ be such that $\left\|\int_{G} d \mu^{b} h_{n}\right\|<\epsilon$ for all $n$, whenever $\|G\|_{\mu}^{b}<\delta$, where $G \in \mathscr{R}$ with $G \subseteq[0, t]$.

Let $N_{0}$ be such that for all $n>N_{0},\left\|G_{n}\right\|_{\mu}^{b_{o}}<\delta / 2$, where

$$
G_{n}=\left\{s \in[0, t]:\left\|h_{n}(s)-\phi(s)\right\| \geq \epsilon / 2\right\} .
$$

Put $G_{n m}=\left\{s \in[0, t]:\left\|h_{n}(s)-h_{m}(s)\right\| \geq \epsilon\right\}$. Then $G_{n m} \in \mathscr{R}$ and $G_{n m} \subseteq G_{n} \cup G_{m}$. Hence

$$
\begin{aligned}
\left\|G_{n m}\right\|_{\mu}^{b}=\left\|G_{n m}\right\|_{\mu}^{b_{o}} & \leq\left\|G_{n}\right\|_{\mu}^{b_{o}}+\left\|G_{m}\right\|_{\mu}^{b_{o}} \\
& \leq \delta / 2+\delta / 2 \\
& =\delta
\end{aligned}
$$

for all $n, m>N_{0}$.

But then, for all $n, m>N_{0}$, we have

$$
\begin{aligned}
\left\|\int_{E} d \mu^{b} h_{n}-\int_{E} d \mu^{b} h_{m}\right\| & \leq\left\|\int_{E \backslash G_{n m}} d \mu^{b}\left(h_{n}-h_{m}\right)\right\|+\left\|\int_{E \cap G_{n m}} d \mu^{b} h_{n}\right\| \\
& +\left\|\int_{E \cap G_{n m}} d \mu^{b} h_{m}\right\| \\
& \leq \epsilon\left\|E \backslash G_{n m}\right\|_{\mu}^{b}+2 \epsilon
\end{aligned}
$$

since $\left\|E \cap G_{n m}\right\|_{\mu}^{b} \leq\left\|G_{n m}\right\|_{\mu}^{b}<\delta$,

$$
\leq \epsilon\left(\|[0, t]\|_{\mu}^{b}+2\right)
$$

The result follows.

It is easy to check that the limit does not depend on the particular approximating sequence $\left(h_{n}\right)$. We shall see that locally square-integrable processes are locally belatedintegrable.

Let $\mathscr{K}_{f}$ be the linear set of (Borel) measurable processes (with respect to $\left\{\chi_{s}(f)\right\}$ ) which are locally square-integrable on $\mathbf{R}^{+}$, equipped with the family of seminorms $\phi \mapsto\left\{\int_{0}^{t}\|\phi(s)\|^{2} d s\right\}^{1 / 2}, t \geq 0$, for $\phi \in \mathscr{K}_{f}$.

Lemma 3.3. For any $\phi \in \mathscr{K}_{f}$ there is a sequence $\left(h_{n}\right)$ of simple processes such that $h_{n} \rightarrow \phi$ in $\mathscr{K}_{f}$.

Proof. For any $\phi \in \mathscr{K}_{f}$ and $\alpha>0$, define

$$
\left(S_{\alpha} \phi\right)(s)= \begin{cases}0, & \text { for } 0 \leq s<\alpha, \\ \phi(s-\alpha), & \text { for } \alpha \leq s\end{cases}
$$


Then $S_{\alpha} \phi \in \mathscr{K}_{f} \quad$ and, for any $t \geq 0, \quad \int_{0}^{t}\left\|s_{\alpha} \phi(s)\right\|^{2} d s \leq \int_{0}^{t}\|\phi(s)\|^{2} d s ; \quad$ also $\int_{0}^{t}\left\|S_{\alpha} \phi(s)-\phi(s)\right\|^{2} d s \rightarrow 0$ as $\alpha \downarrow 0$.

For $n \in \mathbf{N}_{+}$and $k \in \mathbf{N}$, put $I_{n k}=\left[k 2^{-n},(k+1) 2^{-n}\right)$, and let $\mathbb{E}_{n}$ denote the "conditional expectation" corresponding to the partition $\left\{I_{n k}\right\}_{k \in \mathbf{N}}$ of $\mathbf{R}^{+}$;

$$
\left(\mathbb{E}_{n} \phi\right)(s)=2^{n} \int_{I_{n k}} \phi(s) d s \quad\left(s \in I_{n k}, \phi \in \mathscr{K}_{f}\right) .
$$

Then, for any $N \in \mathbb{N}$, and any $\phi \in \mathscr{K}_{f}$,

$$
\int_{0}^{N}\left\|\mathbb{E}_{n} \phi(s)\right\|^{2} d s \leq \int_{0}^{N}\|\phi(s)\|^{2} d s
$$

and $\int_{0}^{N}\left\|\mathbb{E}_{n} \phi(s)-\phi(s)\right\|^{2} d s \rightarrow 0$ as $n \rightarrow \infty$.

Now let $\phi \in \mathscr{K}_{f}$ and define $h_{n}=\chi_{[0, n]} \mathbb{E}_{n} S_{2^{-n}} \phi$. Then $h_{n}$ is a simple process for each $n \in \mathbf{N}_{+}$, and $h_{n} \rightarrow \phi$ in $\mathscr{K}_{f}$.

We can now establish the belated-integrability of the processes in $\mathscr{K}_{f}$.

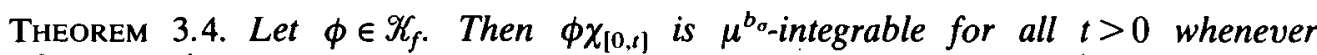

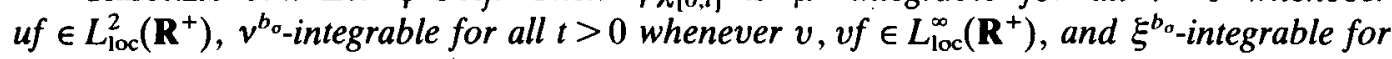
all $t>0$ whenever $f \in L_{\mathrm{loc}}^{\infty}\left(\mathbf{R}^{+}\right)$.

Proof. From the proofs of Theorems 2.1 and 2.2 we have, for simple $h \in \mathscr{K}_{f}, E \in \mathscr{R}$,

$$
\left\|\int_{E} d \eta^{b} h\right\| \leq k_{\eta}(t)\left(\int_{E}\|h\|^{2} d s\right)^{1 / 2}
$$

where $t=\sup E, \eta=\mu, v$ or $\xi$ and

$$
\begin{aligned}
& k_{\mu}(t)^{2}=\int_{0}^{t}|u(s) f(s)|^{2} d s \\
& k_{v}(t)^{2}=e^{t} \sup _{[0, i]}|v|(1+|f|) \\
& k_{\xi}(t)^{2}=e^{t} \sup _{[0, t]}|w|\left(|f|+|f|^{2}\right) .
\end{aligned}
$$

Let $\phi \in \mathscr{K}_{f}$ and, by Lemma 3.3 , let $\left(h_{n}\right)$ be a sequence of simple processes converging to $\phi$ in $\mathscr{H}_{f}$. Then, for any $t \geq 0, h_{n} \rightarrow \phi$ in Lebesgue measure on $[0, t]$ and hence, by Theorems 2.1 and 2.2 , also in $\mu^{b_{o}}, v^{b_{o}}$ and $\xi^{b_{o}}$-measure on $[0, t]$ under the given conditions. Thus it remains only to show that $\left(h_{n}\right)$ is uniformly absolutely continuous with respect to the latter measures on $[0, t]$.

Let $\epsilon>0$ and $t>0$ be given. If $N$ is sufficiently large that

$$
\int_{0}^{t}\left\|h_{n}(s)-h_{m}(s)\right\|^{2} d s<\epsilon^{2}
$$

whenever $n, m \geq N$, and if $M=\max \left\{\left\|h_{k}(s)\right\| ; s \in[0, t], 1 \leq k \leq N\right\}$ then

$$
\left\|\int_{E} d \eta^{b} h_{k}\right\| \leq M\|E\|_{\eta}^{b_{o}} \quad(\eta=\mu, v, \xi)
$$

for $1 \leq k \leq N$ and $E \in \mathscr{R}, E \subseteq[0, t]$. 
On the other hand, from the inequalities (3.1) we have, for $n>N$,

$$
\begin{aligned}
\left\|\int_{E} d \eta^{b} h_{n}\right\| & \leq\left\|\int_{E} d \eta^{b} h_{N}\right\|+\left\|\int_{E} d \eta^{b}\left(h_{n}-h_{N}\right)\right\| \\
& \leq M\|E\|_{\eta}^{b_{o}}+k_{\eta}(t)\left(\int_{E}\left\|h_{n}-h_{N}\right\|^{2} d s\right)^{1 / 2} \\
& <M\|E\|_{\eta}^{b_{\sigma}}+\epsilon k_{\eta}(t) \quad(\eta=\mu, v, \xi) .
\end{aligned}
$$

Thus $\left(h_{n}\right)$ is uniformly absolutely continuous over $[0, t]$ with respect to $\mu^{b_{\sigma}}, v^{b_{\sigma}}$ and $\xi^{b_{\sigma}}$ as required.

The belated integrals of $\phi \in \mathscr{K}_{f}$ are defined to be

$$
\lim _{n} \int_{0}^{t} d \eta^{b} h_{n} \quad(\eta=\mu, v, \xi)
$$

as in Theorem 3.2, and are denoted by

$$
\int_{0}^{t} d a_{u}^{-} \phi, \quad \int_{0}^{t} d a_{v}^{+} \phi, \quad \text { and } \int_{0}^{t} d a_{w}^{0} \phi,
$$

respectively.

4. Quantum stochastic integrals. We shall show, in this section, that the quantum stochastic integrals constructed in [10] can be considered as examples of belated integrals. We recall the definition of operator process [10]. Let $\mathscr{D} \subseteq L^{2}\left(\mathbf{R}^{+}\right) \cap L_{\text {loc }}^{\infty}\left(\mathbf{R}^{+}\right)$be such that $\mathscr{D}$ is dense in $L^{2}\left(\mathbf{R}^{+}\right)$.

Definition 4.1. An operator-valued process is a map $F$ from $\mathbf{R}^{+}$into the set of densely-defined (possibly unbounded) operators in $\mathscr{H}$ such that, for each $f \in \mathscr{D}, \psi(f)$ is in the domain of $F(s)$ for all $s \in \mathbf{R}^{+}$, and $F(s) \psi(f) \in \mathscr{L}_{f}$. F is simple if it has the form $F(s)=\Sigma_{j} F_{j} \chi_{i_{j}}(s)$ for a finite number of bounded intervals $\left\{I_{j}\right\}$ and operators $F_{j}$.

We see immediately that to each operator-valued process $F$ there corresponds a collection of vector-valued processes indexed by $\mathscr{D}$, and conversely. Indeed, for each $f \in \mathscr{D}$, define $\phi^{f}: \mathbf{R}^{+} \rightarrow \mathscr{H}$ by $\phi^{f}(s)=F(s) \psi(f)\left(s \in \mathbf{R}^{+}\right)$. Evidently $\phi^{f}$ is a process with respect to $\left\{\mathscr{X}_{s}(f)\right\}$. Conversely, given a collection $\left\{\phi^{f}: f \in \mathscr{D}\right\}$ such that $\phi^{f}$ is a process with respect to $\left\{\mathscr{Q}_{s}(f)\right\}$, we can define an operator-valued process $F$ by its action on $\{\psi(f): f \in \mathscr{D}\}$ by $F(s) \psi(f)=\phi^{f}(s)$ for $s \in \mathbf{R}^{+} . F(s)$ is a well-defined and densely-defined linear operator since the vectors $\{\psi(f): f \in \mathscr{D}\}$ are linearly independent and span $\mathscr{H}$.

We observe that $\mathscr{X}_{s}(f)=\mathscr{H}_{s} \otimes \psi\left(f^{s}\right)$ where $\mathscr{H}_{s}$ is the symmetric Fock space over $L^{2}([0, s])$ and $f^{s}=f \chi_{(s, \infty)}$ considered as an element of $L^{2}((s, \infty))$. This tensor product decomposition is used in $[9,10,12,13]$.

Let $\mathscr{K}$ denote the set of operator-valued processes $F$ on $\mathbf{R}^{+}$such that $s \mapsto F(s) \psi(f)$ is Borel measurable for each $f \in \mathscr{D}$ and such that $\int_{0}^{t}\|F(s) \psi(f)\|^{2} d s<\infty$ for each $t \geq 0$. Equip the linear space $\mathscr{K}$ with the topology induced by the family of seminorms $F \mapsto\left\{\int_{0}^{t}\|F(s) \psi(f)\|^{2} d s\right\}^{1 / 2}$ indexed by $f \in \mathscr{D}$ and $t \in \mathbf{R}^{+}$.

It was shown in [10] that the elements of $\mathscr{K}$ can be integrated with respect to the Fock creation, preservation and annihilation processes, thus defining quantum stochastic 
integrals as, in general, unbounded operators in $\mathscr{H}$. These operators are defined strongly by their action on the set of exponential vectors $\psi(f)$, for $f \in \mathscr{D}$, thus reducing an operator-integral to an integral of vector-valued processes. We now show that such integrals may be viewed simply as belated integrals.

THEOREM 4.2. For any $F \in \mathscr{K}$, the quantum stochastic integrals $\int F d A_{u}, \int F d \Lambda_{w}$ and, when $v \in L_{\mathrm{loc}}^{\infty}\left(\mathbf{R}^{+}\right)$, also $\int F d A_{v}^{\dagger}$ are all belated integrals in the sense that for any $t>0$ and $f \in \mathscr{D}$, we have

$$
\begin{aligned}
& \int_{0}^{t} F d A_{u}\left(\psi(f)=\int_{0}^{t} d a_{u}^{-} \phi^{f},\right. \\
& \int_{0}^{t} F d \Lambda_{w} \psi(f)=\int_{0}^{t} d a_{w}^{0} \phi^{f}
\end{aligned}
$$

and

$$
\int_{0}^{t} F d A_{v}^{\dagger} \psi(f)=\int_{0}^{t} d a_{v}^{+} \phi^{f}
$$

where $\phi^{f}(s)=F(s) \psi(f)$, for $s \in \mathbf{R}^{+}$.

Proof. If $F \in \mathscr{K}$ then $\phi^{f} \in \mathscr{K}_{f}$ and so, by Theorem 3.4, $\phi^{f}$ is $\mu^{b_{o}}, v^{b_{o}}$ and $\xi^{b_{\sigma}}$-integrable over $[0, t]$ for any $t \geq 0$. Now, for each $f \in \mathscr{D}$, let $\left\{h_{n}^{f}\right\}$ be the sequence of simple processes as constructed in the proof of Lemma 3.3, so that $h_{n}^{f} \rightarrow \phi^{f}$ in $\mathscr{K}_{f}$ as $n \rightarrow \infty$. Since, for given $n$, the $h_{n}^{f}$,s are all piecewise constant on the same intervals, it follows that the formula

$$
F_{n}(s) \psi(f)=h_{n}^{f}(s), \quad \text { for } s \in \mathbf{R}^{+} \text {and } f \in \mathscr{D},
$$

defines a simple operator-valued process $F_{n}$ on $\mathbf{R}^{+}$.

By Lemma 3.3, we see that $F_{n} \rightarrow F$ in $\mathscr{K}$. Now, the quantum stochastic integrals of [10] are constructed via their action on $\{\psi(f): f \in \mathscr{D}\}$ as strong limits of simple integrals. Thus

$$
\int_{0}^{t} F d A_{u} \psi(f)=\lim _{n} \int_{0}^{t} F_{n} d A_{u} \psi(f)
$$

and similarly for the other two integrals. But $\int_{0}^{t} F_{n} d A_{u} \psi(f)$ is precisely the belated integral $\int_{0}^{t} d \mu^{b} h_{n}^{f}$ and, since these converge in $\mathscr{H}_{t}$ to the belated integral $\int_{0}^{t} d a_{u}^{-} \phi^{f}$, equation (4.1) follows. Equations (4.2) and (4.3) follow in the same way.

Remark 4.3. The analysis of [10] includes an "initial space" and considers the one-particle space to the Hilbert space-valued square-integrable (classes of) functions on $\mathbf{R}^{+}$rather than just $L^{2}\left(\mathbf{R}^{+}\right)$. The preceding discussion extends to allow this extra generality with no more than notational changes. We should also note that the definition of a simple map as given in [10] is slightly different from ours. On finite intervals the differences only involve sets of (Lebesgue) measure zero and so are of no consequence to the development of the theory.

REMARK 4.4. By considering measures given by the tensor products $a^{\mp} \otimes 1$ and $1 \otimes a^{\mp}$ on $\mathscr{H} \otimes \mathscr{H}$, it is straightforward to check directly that the quasi-free stochastic 
integrals constructed in $[9,12]$ are belated integrals (one uses the explicit representation on $\mathscr{H} \otimes \mathscr{H}$ as given, for example, in [8]). However, it was shown in [6] that the integrals of [4] are belated integrals, and in [13] it was shown that the integrals of [9] are the same as a class of those constructed in [4].

\section{REFERENCES}

1. D. Appelbaum, The strong Markov property for fermion brownian motion, J. Functional Analysis 65 (1986), 273-291.

2. D. Appelbaum and R. L. Hudson, Fermion Itô's formula and stochastic evolutions, Commun. Math. Phys. 96 (1984), 473-496.

3. C. Barnett, R. F. Streater and I. F. Wilde, The Itô-Clifford integral, J. Functional Analysis 48 (1982), 172-212.

4. C. Barnett, R. F. Streater and I. F. Wilde, Quasi-free quantum stochastic integrals for the CAR and CCR, J. Functional Analysis 52 (1983), 19-47.

5. C. Barnett, R. F. Streater and I. F. Wilde, Quantum stochastic integrals under standing hypotheses, J. Math. Analysis and Applications 127 (1987), 181-192.

6. C. Barnett and I. F. Wilde, Belated integrals, J. Functional Analysis 66 (1986), 283-307.

7. R. G. Bartle, A general bilinear vector integral, Studia Math. 15 (1956), 337-352.

8. G. F. Dell'Antonio, Structure of the algebras of some free systems, Commun. Math. Phys. 9 (1968), 81-117.

9. R. L. Hudson and J. M. Lindsay, A non-commutative martingale representation theorem for non-Fock quantum Brownian motion, J. Functional Analysis 61 (1985), 202-221.

10. R. L. Hudson and K. R. Parthasarathy, Quantum Itô's formula and stochastic evolutions, Commun. Math. Phys. 93 (1984), 901-923.

11. R. L. Hudson and K. R. Parthasarathy, Unification of fermion and boson stochastic calculus, Commun. Math. Phys. 104 (1986), 457-470. 1985).

12. J. M. Lindsay, A quantum stochastic calculus, Ph.D. thesis (University of Nottingham

13. J. M. Lindsay and I. F. Wilde, On non-Fock boson stochastic integrals, J. Functional Analysis 65 (1986), 76-82.

Chris Barnett

Department of Mathematics

IMPERIAL COLlege OF SCIENCE

TeChNology and Medicine

LONDON SW7 2BZ

J. M. LINDSAY

Department of Mathematics

UNIVERSITY OF NOTTINGHAM

UNIVERSITY PARK

NotTINGHAM NG7 2RD
Ivan F. Wilde

Department of Mathematics

King's College

STRAND

LONDON WC2R 2LS 